\title{
The hard X-ray response of a CdZnTe ring-drift detector
}

\author{
A.Owens, R.den Hartog, F. Quarati \\ Science Payloads and Advanced Concepts Office, SCI-A, \\ European Space Agency, ESTEC, Noordwijk, The Netherlands \\ V. Gostilo, V. Kondratjev, A. Loupilov \\ Baltic Scientific Instruments, 26 Ganibu dambis, PO Box 33, Riga LV-1005, Latvia \\ A.G.Kozorezov, J.K.Wigmore \\ Department of Physics, Lancaster University, Lancaster, UK
}

A. Webb, E. Welter

HASYLAB at DESY, Notkestrasse 85, D 22607 Hamburg, Germany

(Dated: May 8, 2007)

We present the results of an experimental study of a new type of CdZnTe detector of hard X- and $\gamma$-rays - a ring-drift detector. The device consists of a double ring electrode structure surrounding a central point anode with a guard plane surrounding the outer anode ring. The detector can be operated in two distinctively different modes of charge collection -pseudo Hemispherical and pseudo Drift. We study the detector response profiles obtained by scanning the focused x-ray beam over the whole detector area, specifically the variations in count rate, peak position and energy resolution for X-rays from 10 to $100 \mathrm{keV}$. In addition, at $662 \mathrm{keV}$ the energy resolution was shown to be $4.8 \mathrm{keV}$, more than factor 2 better than for CdZnTe coplanar grid detectors. To interpret the experimental data, we derive an analytical expression for the spatial distribution of the electric field inside the detector and neglecting carrier diffusion, and identify carrier collection patterns for both modes of operation within the drift model approximation. We show that this model provides a good understanding of measured profiles. 


\section{INTRODUCTION}

For conventional radiation detectors fabricated from compound semiconductors, the wide disparity between the transport properties of the electron and holes means that detector performance is limited by the carrier with the poorest mobility-lifetime product ${ }^{1,2}$. This effectively limits maximum detector thicknesses for spectroscopy applications, resulting in a trade between spectroscopic performance and dynamic range. Significant improvement in spectral acuity can be achieved by various methods aimed at minimizing effects of carriers with poor transport properties. Such methods, which are often called single polarity sensing techniques (although in some of the best known examples the carriers with poor mobility-lifetime product still control the improved spectral resolution), have been widely applied to CdZnTe detectors to overcome poor hole transport ${ }^{1-3}$. Examples include hemispherical and co-planar grid geometries. In silicon technology, which is far more advanced, a considerable reduction in noise can be made by shaping the electric field and channelling charge to a small central read-out anode with a capacitance which is much smaller than that of the active surface area. In a drift device ${ }^{4}$, this is achieved using a number of concentric ring electrodes designed and biased so that potential gradient induces a transverse electric field pushing electrons towards

the central anode. In this paper, we describe a similar approach applied to a CdZnTe detector. Our motivation is centered not on achieving low readout noise, but on channelling electrons directly to the read-out node whilst incurring minimum charge loss. At the same time, holes drift to their collecting electrodes further away from the near field region of the central anode. This results in their contributions to a detector response being greatly diminished, making the detector essentially a single carrier sensing device. An extra benefit of this design is that by the proper adjustment of the ring potentials, the detector may be operated in several modes of charge collection of which pseudo Hemispherical and Drift modes are explored in this paper.

\section{EXPERIMENTAL}

The detector is fabricated on a single CdZnTe crystal of size $5 \times 5 \times 1 \mathrm{~mm}^{3}$. On the top face, the crystal is patterned by evaporating gold electrodes consisting of a small circular anode, a double ring electrode structure followed by a guard ring. A plane cathode is evaporated on the backside. The detector geometry is shown schematically in Fig. 1. The central electrode is used for charge collection and the two ring electrodes $R_{1}$ and $R_{2}$ and the guard ring $G R$ are used to shape the electric field in the crystal. The chip is bonded to a ceramic substrate, which is coupled to a two-stage Peltier cooler capable of cooling the detector to $-20^{\circ} \mathrm{C}$. To ensure low noise, the preamplifier input $\mathrm{FET}$ is 


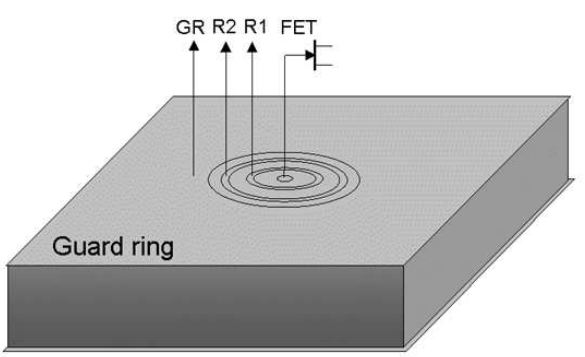

FIG. 1: Schematic image of our ring detector. The crystal has dimensions $5 \times 5 \times 1 \mathrm{~mm}^{3}$. The inner anode has a diameter of 80 $\mu \mathrm{m}$, and mean radii of the other two concentric ring electrodes are 0.19 and $0.39 \mathrm{~mm}$ respectively. The guard ring $G R$ covers the remainder of the surface beyond a radius of $0.59 \mathrm{~mm}$.

TABLE I: The biasing schemes for Hemispherical and Drift modes

\begin{tabular}{|c|c|}
\hline Hemispherical mode & Drift mode \\
\hline \hline$V_{\text {anode }}=0 \mathrm{~V}$ & $V_{\text {anode }}=0 \mathrm{~V}$ \\
\hline$V_{R_{1}}=-52.3 \mathrm{~V}$ & $V_{R_{1}}=-15.0 \mathrm{~V}$ \\
\hline$V_{R_{2}}=-60.7 \mathrm{~V}$ & $V_{R_{2}}=-17.2 \mathrm{~V}$ \\
\hline$V_{G R}=-47.9 \mathrm{~V}$ & $V_{G R}=-138 \mathrm{~V}$ \\
\hline$V_{\text {cathode }}=-136.5 \mathrm{~V}$ & $V_{\text {cathode }}=-46.2 \mathrm{~V}$ \\
\hline
\end{tabular}

also mounted on the substrate, close to the detector. For the measurements described here, the detector temperature was set at $+8^{\circ} \mathrm{C}$. Contacting to the electrodes is achieved by wire bonding. The cooler/substrate/chip assembly is packaged in an hermetically sealed TO8-style aluminium housing. The detector is viewed through a thin beryllium window. The rest of the analog chain consists of an externally mounted resistive feedback preamplifier followed by an ORTEC 671 spectroscopy amplifier. A shaping time of $3 \mu$ s was found to be optimum. In Section 3 we will describe experimental results taken with the detector operated in both Hemispherical and Drift modes. The biasing schemes which were used are listed in Table 1 . The device was initially characterized in both modes using radioactive calibration sources $\left({ }^{55} \mathrm{Fe},{ }^{241} \mathrm{Am},{ }^{133} \mathrm{Ba}\right.$ and $\left.{ }^{137} \mathrm{Cs}\right)$ under full illumination. Both modes of operation show excellent energy resolution over a wide dynamic range. The FWHM detector energy resolutions are plotted in Fig. 2 and are well fit by linear functions. From the figure, we see that on average, the Drift mode gives $\sim 0.3$ keV better energy resolution across the entire dynamic range, from $0.7 \mathrm{keV} \mathrm{FWHM} \mathrm{at} 5.9 \mathrm{keV}$ to $5 \mathrm{keV}$ FWHM at $662 \mathrm{keV}$, which is a factor of 2 better than achieved for coplanar grid configuration ${ }^{3}$. However, although energy resolution is better in Drift mode, the peak-to-background ratios are larger in Hemispherical mode. In Section 4 we will analyze the 


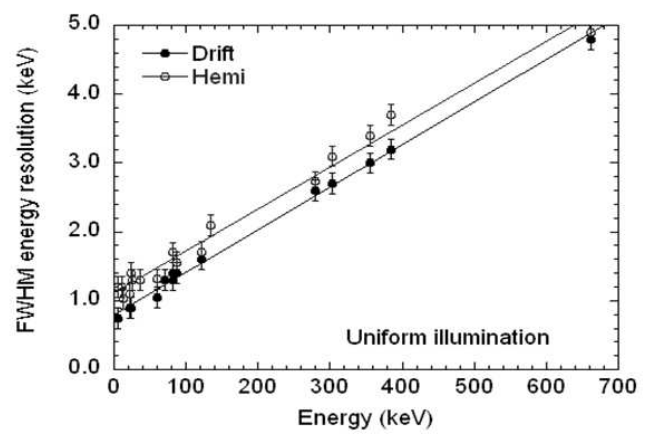

FIG. 2: The measured FWHM energy resolution in both Hemispherical and Drift mode configurations. The data were taken under full area illumination using radioactive calibrations sources. Across the entire energy range, the resolutions of the Drift mode are $\sim 0.3 \mathrm{keV}$ better than for the Hemispherical mode

dependence of carrier collection patterns on biasing, and we show that there exists a clear intrinsic difference between the two modes of operation.

\section{SYNCHROTRON X-RAY MEASUREMENTS}

Synchrotron beam X-ray measurements were carried out at the X-1 beam line at the Hamburger Synchrotronstrahlungslabor (HASYLAB) radiation facility in Hamburg, Germany. This beam line utilizes a double Si crystal monochromator to produce highly monochromatic X-ray beams across the energy range $10.5 \mathrm{keV}^{\text {to }} 100 \mathrm{keV}$. To achieve such a large energy range, a [511] reflection is used, yielding an intrinsic energy resolution of 1 eV at 10 $\mathrm{keV}$ rising to $20 \mathrm{eV}$ at $100 \mathrm{keV}$. The beam spot size is defined by a pair of precision stepper-driven slits, positioned immediately in front of the detector. The beam line employs a DMOSTAB (Digital MOnochromater STABilizer) servo loop system to ensure constant beam intensity at the detector. For the majority of the measurements described here, a slit size of $50 \times 50 \mu \mathrm{m}^{2}$ was used. The detector was mounted on an X-Y stage capable of positioning the array to a precision of $<1 \mu \mathrm{m}$ along each axis.

\section{A. Spatial response}

During initial scanning of the detector to locate its boundaries prior to measurement, it became apparent that the spatial distribution of events was not uniform as originally expected, both in Hemispherical and Drift modes. This is shown in Fig. 3 in which we plot a raster scan of a $50 \times 50 \mu \mathrm{m}^{2}, 20 \mathrm{keV}$ beam across the detector. The step size is 50 $\mu \mathrm{m}$. Spectra were accumulated at each position for a fixed time interval. The total count rate above a $3 \mathrm{keV}$ threshold, 


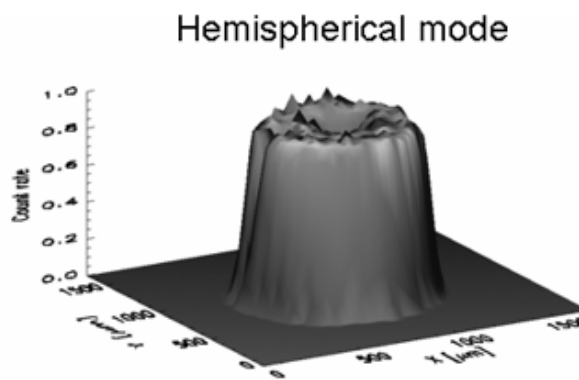

(a)
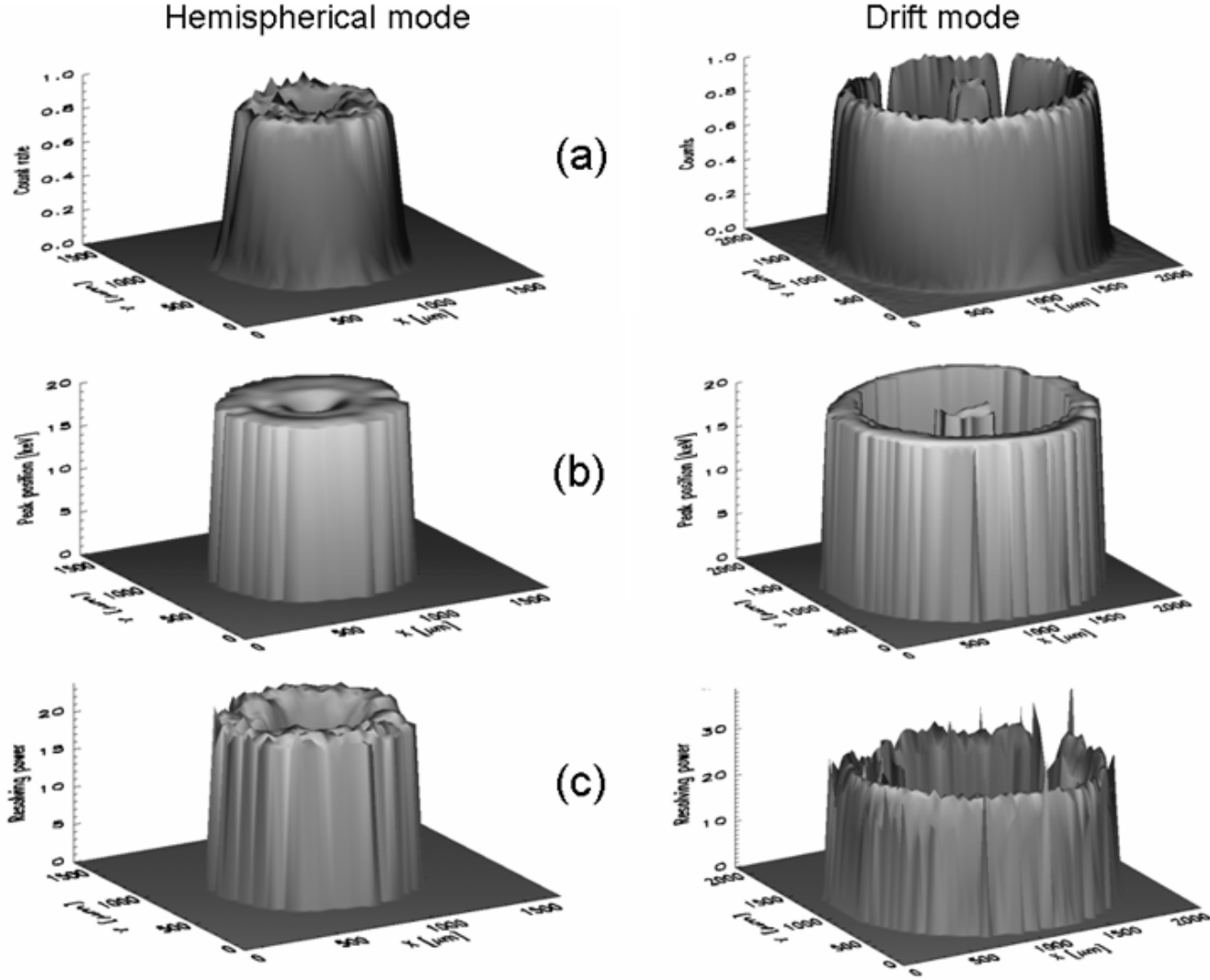

FIG. 3: The spatial responses of the detector, mapped at HASYLAB using a $50 \times 50 \mu \mathrm{m}^{2}, 20 \mathrm{keV}$ X-ray beam. Both the Hemispherical (left) and Drift modes (right) have been mapped. (a) shows the variation in count rate profile, (b) the fitted centroid of the photo peak, (i.e., gain) and (c) the resolving power, i.e., E/ $\Delta \mathrm{E}$. These distributions were derived from spectra accumulated at 1600 positions across the detector. Note, the difference in lateral size between the Hemispherical and Drift modes

the peak centroid position (i.e., the gain) and the FWHM energy resolution were determined by best-fitting. The results are shown in Fig. 3 for each of the recorded parameters. These data comprise approximately 1600 individual spectra. The energy resolution is characterized in terms of detector resolving power (i.e. $\mathrm{E} / \Delta \mathrm{E}$ ) to ensure numerical stability across the detector boundaries (i.e. the function goes to zero outside the active area). From the figure, we see that while the spatial response in the Hemispheric mode is circular and reasonably flat with a slight depression at the center, the response in Drift mode consists of an outer "ring of events" surrounding a circular enhancement around the anode at the center. In Fig. 4, we plot both distributions in the form of contour plots and overlaid the mask used to produce the electrode structure. We see that the spatial distribution of events in the Hemispherical mode extends to the outer radius of ring $R_{2}$ while the response of the detector in Drift mode consists of two components, an 

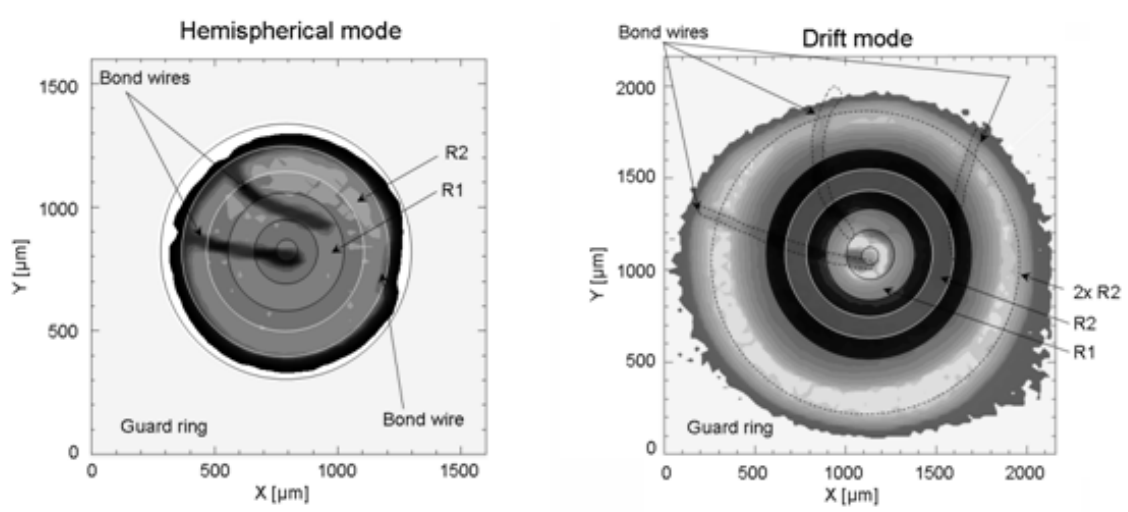

FIG. 4: Contours of the count rate, spatially mapped in both Hemispherical and Drift modes. We have overlaid the mask pattern showing the position of the anode, ring electrodes and guard ring. The absorption of photons in the bond wires can clearly be seen in both modes

inner circular distribution of events which extends to the outer radius of the ring $R_{1}$, surrounded by a narrow "ring of events" inside the guard ring at approximately $2 \times R_{2}$. No further "event rings" were detected in extended scans out to a distance of $3.5 \mathrm{~mm}$. Very few events were detected in the region between the "event ring" at $2 R_{2}$ and the inner enhancement at $R_{1}$, In fact the events in the gap constitute $\simeq 0.3 \%$ of the total recorded events. This effect depends on photon energy as is illustrated in Fig. 5, in which we show a scan in the $X$-direction at $Y=0$ for photons of different energies. The beam size is $20 \times 20 \mu \mathrm{m}^{2}$ and the step size is $20 \mu \mathrm{m}$. From this figure, it can be seen that at low photon energy the gap between the "event ring" at $0.8 \mathrm{~mm}$ and the central enhancement at $X=0 \mathrm{~mm}$ is almost devoid of events. However as photon energy increases, the gap begins to fill-in, becoming comparable in amplitude to the "event ring" at the highest energies. The response near $X=0$ is also a factor of two larger at the highest energies, while it is generally comparable in amplitude with the ring at low energies. This is all consistent with the picture of charge transport depending on the depth of the photon absorption site where the carriers are initially generated.

\section{B. Energy loss spectra}

Because of the peculiar spatial response of the detector, spectra were accumulated at $Y=0$ and at $X=R_{2}$ in the Hemispherical mode and $X=2 R_{2}$ in the Drift mode (see Fig. 6). In Figs. 6a and 6b, we show a composite of energy-loss spectra recorded at these coordinates over the energy range $10.5 \mathrm{keV}$ to $100 \mathrm{keV}$. In Drift mode, we note that spectra taken within the inner circular response zone $\left(X=R_{1}\right)$ were of much lower quality than those taken at the "ring" and are therefore not shown here. From Fig. 6, we see that the photopeaks are clearly resolved in both 


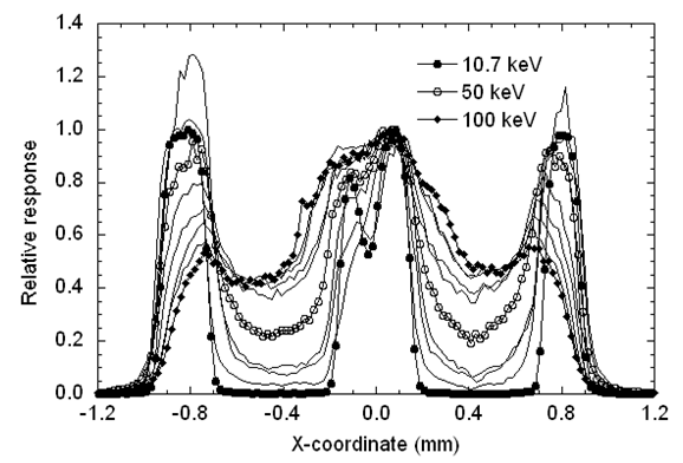

FIG. 5: Scan along the $X$-axis at $Y=0$ at different photon energies of the detector in Drift mode. In this case, the beam size is $20 \times 20 \mu \mathrm{m} 2$ and the step size is $20 \mu \mathrm{m}$. The dip in response near $X=0$ is due to absorption in the anode bond wire

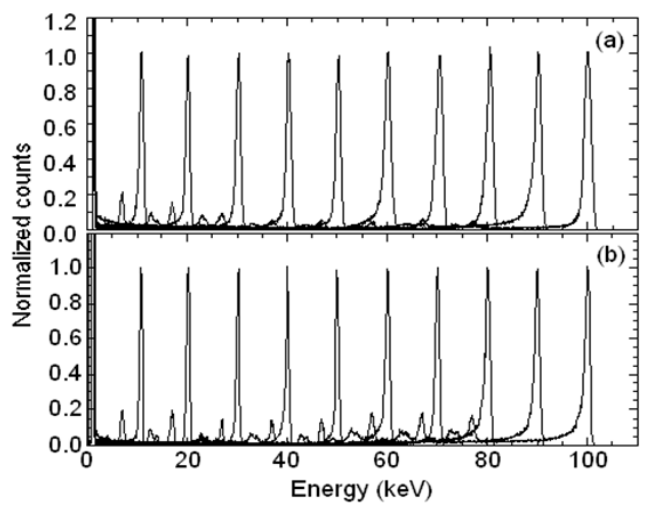

FIG. 6: Composite of energy loss spectra measured at $Y=0$ at the peak response along the $X$-axis for both the Hemispherical (a) and Drift (b) modes. For (a) the response was measured at $X=R_{2}$ and for (b) at $X=2 R_{2}$

modes. The line shape is significantly asymmetric even at low energies. The noise threshold (defined to be the lowest energy for which the low energy noise attains an amplitude of 0.5 of the photo peak) is $0.5 \mathrm{keV}$. In Fig. 7 we show the detector proportionality of response or linearity curve for both modes.

The data are plotted in the form of relative peak position to accentuate deviations form linearity. The lower panel shows the corresponding residuals, i.e., the percentage deviation from a best linear fit. From the figure, we see that for the Hemispherical mode the detector is linear showing no deviations in the residuals. However, this is not true for the Drift mode where we see a clear departure from linearity at energies $<50 \mathrm{keV}$. 


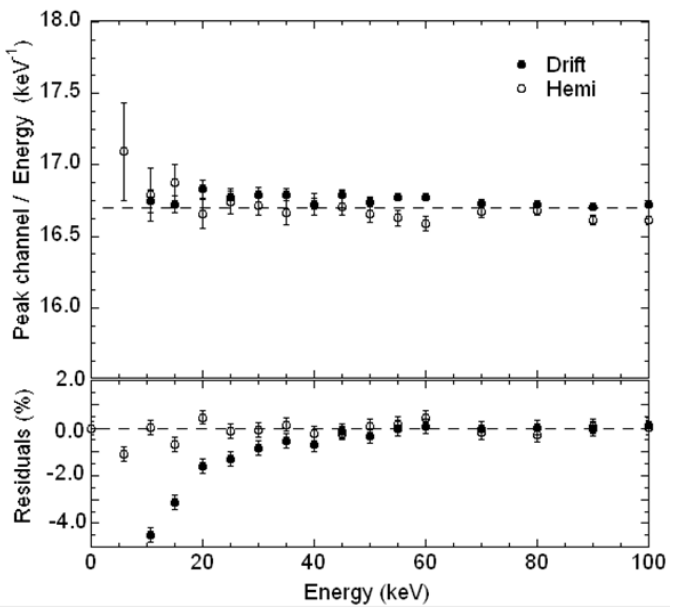

FIG. 7: The detector proportionality of response or linearity curve for the both modes. The data are plotted in the form of centroid ADC channel divided by energy versus energy to accentuate structure. The lower panel shows the corresponding residuals, i.e., the percentage deviation from a best linear fit (measured peak channel-calculated peak channel /calculated peak channel $\times 100)$

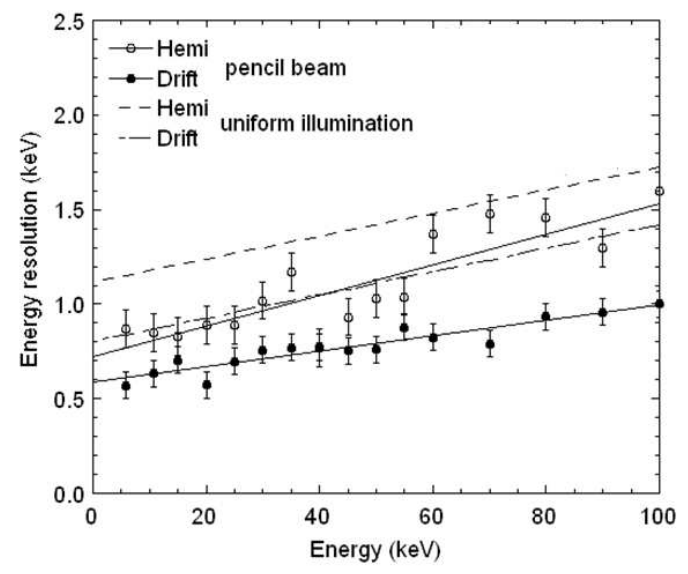

FIG. 8: The measured FWHM energy resolution obtained for Hemispherical and Drift modes. For the Hemispherical mode, the spectra were taken at the radial distance of the $R_{2}$ ring, while for the Drift mode they were taken at $2 R_{2}$. For comparison, we also show the resolutions measured under uniform illumination

\section{Energy resolution}

The FWHM energy resolutions for each mode were determined from the pulse height data shown in Fig. 6 and plotted in Fig. 8. The measured resolutions $(\Delta \mathrm{E} / \mathrm{E})$ under pencil beam illumination were $6.3 \%$ (Drift mode) and $8.1 \%$ (Hemispherical mode) at $10.5 \mathrm{keV}$, and $1.4 \%$ (Drift mode) and $2.0 \%$ (Hemispherical mode) at $60 \mathrm{keV}$. For full area illumination, the measured FWHM's are slightly worse (see Fig. 8). 
At $60 \mathrm{keV}$ they are 2.0\% (Drift mode) and 2.5\% (Hemispherical mode). The detector was also found to maintain good spectral resolution at gamma-ray energies. For example, at $662 \mathrm{keV}$, the FWHM energy resolutions were $0.73 \%$ (Drift mode) and $0.79 \%$ (Hemispherical mode). A sum of all of the $20 \mathrm{keV}$ spectra obtained during the raster scans shown in Fig. 3 yielded energy resolutions that were only $\sim 140 \mathrm{eV}$ worse than the single point "ring" measurements for both the Hemi- and Drift modes. This indicates that, although the spatial distribution of events may not look uniform, the spectra largely are. Note, that for the Drift mode, spectra taken at the inner ring $R_{1}$ (see Fig. 6), gave a wide dispersion in energy resolutions - between 1 and 6 keV FWHM across the entire energy range.

\section{CARRIER COLLECTION PATTERNS}

In order to interpret the experimental results we need to model the distribution of electrostatic fields in the detector. For the geometry of the detector shown in Fig. 1 we are interested in the distribution of electrostatic fields in the central region well away from the edges of the crystal. Therefore we will consider for the model that the guard ring extends to infinity. Since the system possesses cylindrical symmetry, the electrostatic potential is a function only of the radial distance from the center and the coordinate in the normal direction along $z$-axis, thus $\Phi=\Phi(r, z)$. Inside the detector and in the vacuum $\Delta \Phi=0$. Boundary conditions are $\Phi(r, 0)=0$ at the cathode plane, whilst at the plane $z=L$ (where $L$ is the detector thickness)

$$
\Phi(r, L)= \begin{cases}V_{c} & : r \leq r_{c} \\ V_{1} & : \quad r_{11} \leq r \leq r_{12} \\ V_{2} & : \quad r_{21} \leq r \leq r_{22} \\ V_{G} & : \quad r \geq r_{G}\end{cases}
$$

Here $r_{c}$ is the radius of the central anode, $r_{11}, r_{12}$ and $r_{21}, r_{22}$ are the inner and outer radii for the first and second rings respectively, and $r_{G}$ is the inner radius of the guard ring. At the gaps between the electrodes at the anode plane we need to introduce the boundary conditions with special care. It is well known that surface resistivity is an important parameter of the CZT detectors ${ }^{6-9}$. It results in high leakage currents, and also affects electric field distribution near the contacts. Keeping the model as simple as possible we assume that there exists a shallow surface layer of significantly smaller resistivity than the bulk CZT material so that in a stationary regime surface currents flow between the electrodes, whilst bulk contributions can be neglected, and hence model the variation of electrostatic potential in the gaps between electrodes to be consistent with continuity of the surface current. These assumptions introduce major limitations to the validity of the results. We will discuss their implications below. 
If we designate the resulting potential $V(r)$, then solving the standard Dirichlet problem for electrostatic potential in cylindrical coordinates

$$
\begin{aligned}
\frac{\partial^{2} \Phi}{\partial z^{2}}+\frac{\partial^{2} \Phi}{\partial r^{2}}+\frac{1}{r} \frac{\partial \Phi}{\partial r} & =0: \\
\Phi(r, z=0) & =0 \\
\Phi(r, z=L) & =V(r)
\end{aligned}
$$

we obtain

$$
\Phi(r, z)=V_{G} z+\int_{0}^{\infty} \mathrm{d} t^{\prime} \frac{t^{\prime} \sinh z t^{\prime}}{\sinh t^{\prime}} J_{0}\left(r t^{\prime}\right) \int_{0}^{r_{G}} \mathrm{~d} r^{\prime} r^{\prime} J_{0}\left(r^{\prime} t^{\prime}\right)\left[V\left(r^{\prime}\right)-V_{G}\right]
$$

where $J_{0}(x)$ is the Bessel function. Continuity of the surface current $j_{s}(r)$ requires that $\operatorname{div}\left(j_{s}(r)\right)=0$. Therefore $\partial\left(r j_{s}\right) / \partial r=0$, hence $j_{s}$ is proportional to $1 / r$, leading to a logarithmic variation in $V(r)$ in the gaps. The potentials must be continuous at $r_{c}, r_{11}, r_{12}, r_{21}, r_{22}$ and $r_{G}$, where they must coincide with the values given by (1). As a result we obtain

$$
V(r)=\left\{\begin{aligned}
V_{c} & : \quad r \leq r_{c} \\
{\left[V_{c} \ln \left(r_{11} / r\right)+V_{1} \ln \left(r / r_{c}\right)\right] / \ln \left(r_{11} / r_{c}\right) } & : \quad r_{c} \leq r \leq r_{11} \\
V_{1} & : \quad r_{11} \leq r \leq r_{12} \\
{\left[V_{1} \ln \left(r_{21} / r\right)+V_{2} \ln \left(r / r_{12}\right)\right] / \ln \left(r_{21} / r_{12}\right) } & : \quad r_{12} \leq r \leq r_{21} \\
V_{2} & : \quad r_{21} \leq r \leq r_{22} \\
{\left[V_{2} \ln \left(r_{G} / r\right)+V_{G} \ln \left(r / r_{22}\right)\right] / \ln \left(r_{G} / r_{22}\right) } & : \quad r_{22} \leq r \leq r_{G} \\
V_{G} & : \quad r \geq r_{G}
\end{aligned}\right.
$$

Equipotentials, calculated with the use of (3) for the Hemispherical mode with the biasing as given in the Table 1, are shown in Fig.9a. As expected the equipotentials show that on approach to the $z=L$ plane, the electric field distribution becomes non-uniform with a saddle point formed opposite the outer ring. For the Drift mode, the equipotentials are shown in Fig.10a. It is seen that the topology of the equipotential surfaces is completely different from the hemispheric mode. A saddle point of the electrostatic potential is located beneath the central anode approximately at half the depth of the detector. The exact position depends on biasing in the Drift mode and can be varied in the vertical direction. The features of the electrostatic potential shown in Figs. 9a and 10a define the drift trajectories for both carrier types. If carrier diffusion is neglected, then the carrier collection pattern can be easily deduced from the field lines. Figs. $9 \mathrm{~b}$ and $10 \mathrm{~b}$ show schematically the electron collection zones (light regions) 


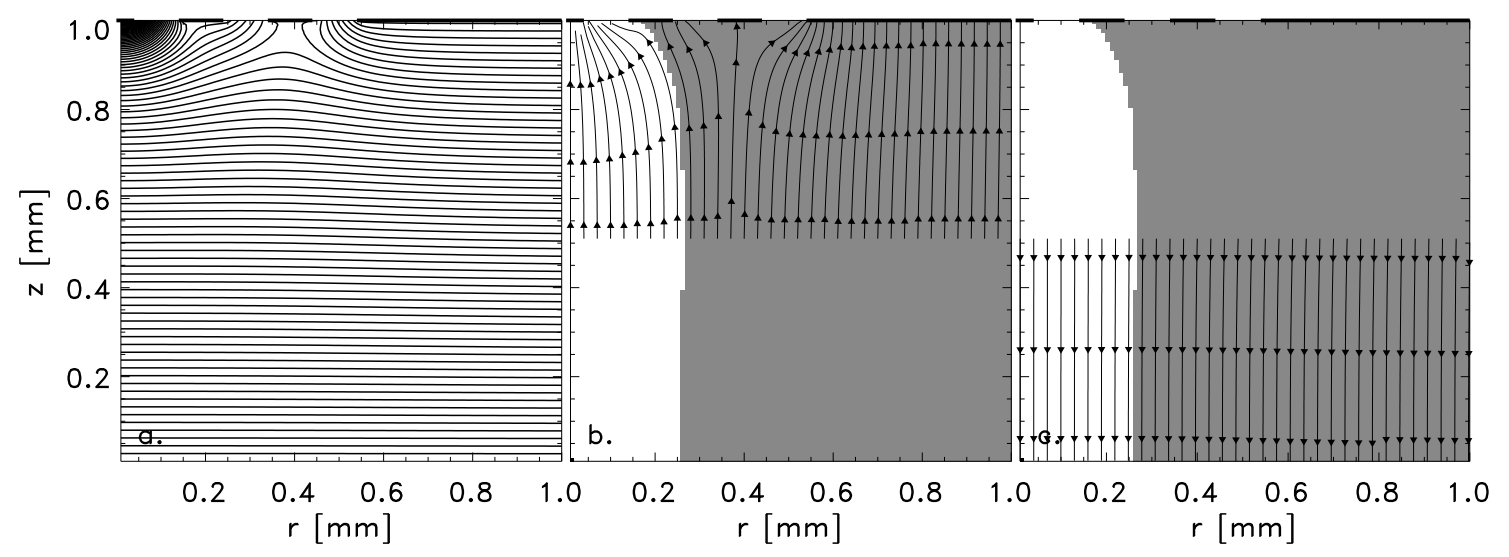

FIG. 9: Hemispherical mode. (a) equipotential lines (b) electron collection pattern and examples of electron trajectories and (c) examples of hole trajectories starting from the same initial positions. In the electron collection pattern the light region indicates initial positions from which the collecting anode will be reached, and the grey region initial positions from which the electrons will reach the top surface outside the collecting anode.

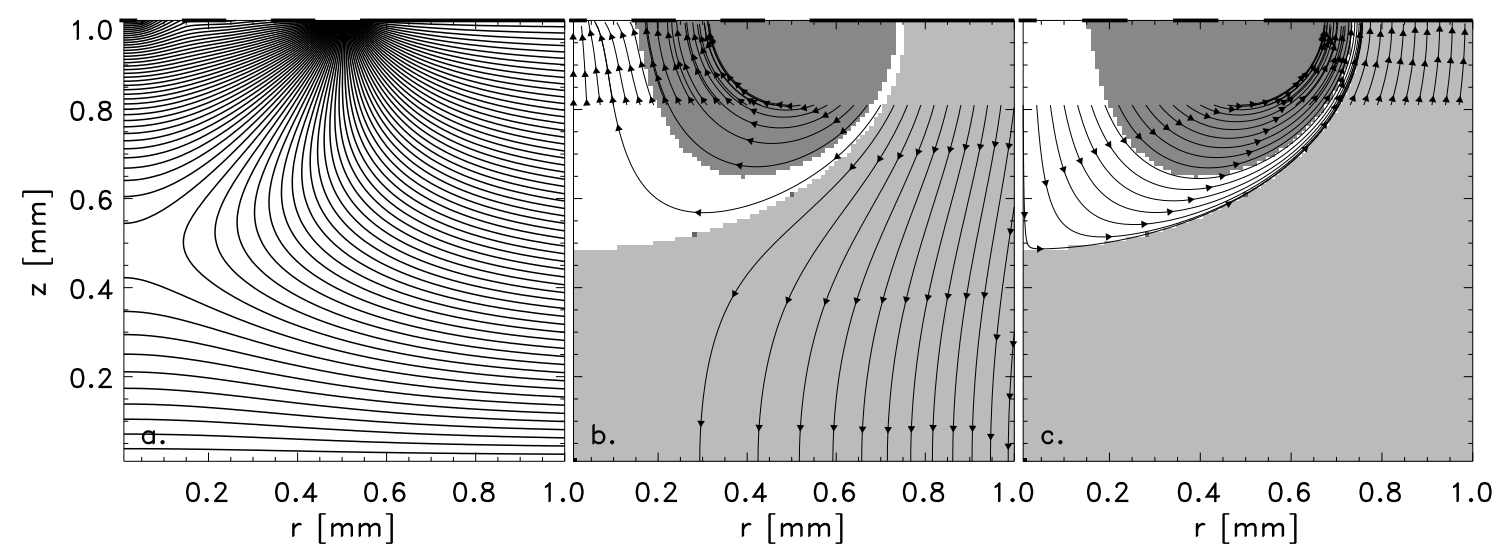

FIG. 10: Drift mode. (a) equipotential lines (b) electron collection pattern and examples of electron trajectories and (c) examples of hole trajectories starting from the same initial positions. As in Fig.9, the light region comprises initial positions from which the collecting anode will be reached, and the light grey area initial positions from which the electrons will end on the cathode surface, and the dark grey area indicates the 'dead zone', containing initial positions from which the top surface outside the collecting anode will be reached.

and sample electron trajectories for both modes, while the hole collection trajectories are illustrated in Figs. 9c and $10 \mathrm{c}$.

It is seen that for the Hemispherical mode the electron collection region comprises most of the detector volume inside the cylinder $0<z \leq L, r \leq r_{21}$, excluding the parts close to the saddle point of the electrostatic potential. Electrons, which are created in the latter region are instead collected by either the inner or the guard ring. Holes 
will move in the opposite direction, and in all situations move towards either the cathode or the outer ring. If the detector is operated in the Drift mode, then the electrons from the light area will move towards the central anode. Those generated inside the grey region will move towards the rings or the cathode. Holes from all areas will move towards the guard ring.

\section{DETECTOR RESPONSE}

A general formula for the charge collected by any electrode can be written as ${ }^{10}$

$$
Q\left(t, \mathbf{x}_{a}\right)=-\int_{S} \mathrm{~d}^{2} \mathbf{r}^{\prime} \int_{0}^{t} \mathrm{~d} t^{\prime} j_{z}\left(\mathbf{r}^{\prime}, L, t^{\prime}\right)-\int_{\Omega} \mathrm{d} \mathbf{x}^{\prime} \Phi_{w}\left(\mathbf{x}^{\prime}\right) \rho\left(\mathbf{x}^{\prime}, t\right)
$$

Here $\rho\left(\mathbf{x}^{\prime}, t\right)=\rho_{e}\left(\mathbf{x}^{\prime}, t\right)+\rho_{h}\left(\mathbf{x}^{\prime}, t\right)$ and $j\left(\mathbf{x}^{\prime}, t\right)=j_{e}\left(\mathbf{x}^{\prime}, t\right)+j_{h}\left(\mathbf{x}^{\prime}, t\right)$ are charge and current density as a functions of coordinates $\mathbf{x}^{\prime}$ and time $t$ respectively, both assumed to be equal to zero at $t=0$, and $\mathbf{x}_{a}$ is the coordinate of absorption site. The subscripts $(e, h)$ here and elsewhere label the contributions from electrons and holes respectively, $\Phi_{w}(\mathbf{x})$ is the weighting potential for the electrode, $S$ is its area, and $\Omega$ is the volume of the detector. In the drift approximation, by neglecting carrier diffusion and solving the charge continuity equations we find

$$
\rho_{e, h}(\mathbf{x}, t)=q_{e, h} \Theta(t) N_{0} \exp \left(-t / \tau_{e, h}\right) \delta\left(\mathbf{x}-\mathbf{x}_{e, h}\left(t, \mathbf{x}_{a}\right)\right)+q_{e, h} \int_{0}^{t} \frac{\mathrm{d} t^{\prime}}{\tau_{e, h}} \exp \left(-\frac{t^{\prime}}{\tau_{e, h}}\right) \delta\left(\mathbf{x}-\mathbf{x}_{e, h}\left(t^{\prime}, \mathbf{x}_{a}\right)\right)
$$

where $q_{e, h}$ is the carrier charge, $\Theta(t)$ is the Heaviside function, $N_{0}$ is the number of e-h pairs generated at the moment of photon absorption $t=0, \tau_{e, h}$ are electron and hole lifetimes, and $\mathbf{x}_{e, h}\left(t, \mathbf{x}_{a}\right)$ are carrier drift trajectories which originate at the absorption site with the coordinate $\mathbf{x}_{a}$. In the drift approximation $\mathbf{x}_{e, h}\left(t, \mathbf{x}_{a}\right)$ satisfies the equations

$$
\dot{\mathbf{x}}_{e, h}=-\mu_{e, h} \nabla \Phi(\mathbf{x})
$$

For a ring detector we obtain

$$
Q=Q(t \rightarrow \infty)=-\int_{r<r_{c}} \mathrm{~d}^{2} \mathbf{r}^{\prime} \int_{0}^{\infty} \mathrm{d} t^{\prime} j_{e, z}\left(\mathbf{r}^{\prime}, L, t^{\prime}\right)-\int_{\Omega} \mathrm{d} \mathbf{x}^{\prime} \Phi_{w}\left(\mathbf{x}^{\prime}\right) \rho\left(\mathbf{x}^{\prime}, t\right)
$$

Taking $j_{e, z}(\mathbf{x}, t)=q_{e} N_{0} \exp \left(-t / \tau_{e, h}\right) \delta\left(\mathbf{x}-\mathbf{x}_{e, h}\left(t, \mathbf{x}_{a}\right)\right) \mu_{e} E_{z}(\mathbf{x})$, where $\mu_{e}$ is electron mobility and $E_{z}(\mathbf{x})$ is the $z$ component of the electric field at a point $\mathbf{x}$ and performing the integrations in (8) we obtain

$Q=Q\left(\mathbf{x}_{a}\right)=-e N_{0} \exp \left(-\frac{t^{*}\left(\mathbf{x}_{a}\right)}{\tau_{e}}\right)-e N_{0} \int_{0}^{\infty} \mathrm{d} t^{\prime}\left[\frac{1}{\tau_{e}} \exp \left(-\frac{t^{\prime}}{\tau_{e}}\right) \Phi_{w}\left(\mathbf{x}_{e}\left(t^{\prime}, \mathbf{x}_{a}\right)\right)-\frac{1}{\tau_{h}} \exp \left(-\frac{t^{\prime}}{\tau_{h}}\right) \Phi_{w}\left(\mathbf{x}_{h}\left(t^{\prime}, \mathbf{x}_{a}\right)\right)\right](9)$

Here $t^{*}\left(\mathbf{x}_{a}\right)$ is the time for an electron to reach the central anode moving along its drift trajectory $\mathbf{x}_{e}\left(t, \mathbf{x}_{a}\right)$. The first term in (9) describes the contribution to the detector response from carriers reaching the anode without being trapped, while the integral term arises for contributions from trapped electrons and holes. 


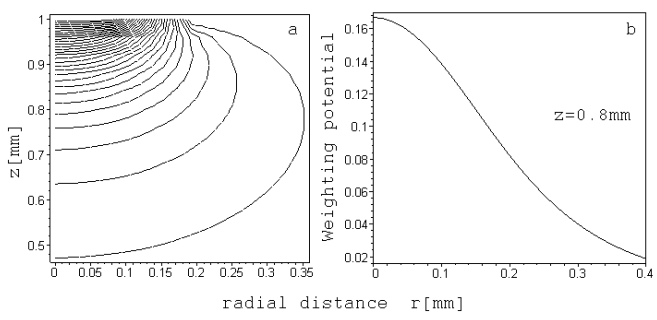

FIG. 11: Weighting potential for the central anode of a ring detector. a) contour plot, b) Variation of $\Phi_{w}$ with radial distance at a depth $z=0.8 \mathrm{~L}$

Finally, the explicit expression for the weighting potential $\Phi_{w}(\mathbf{x})$ follows from (3). Indeed from the definition for the weighting potential, taking the potential on the anode plane as given in (4) but with $V_{c}=1, V_{1}=V_{2}=V_{G}=0$, we obtain

$$
\Phi_{w}(r, z)=\int_{0}^{\infty} \mathrm{d} t^{\prime} \frac{t^{\prime} \sinh z t^{\prime}}{\sinh t^{\prime}} J_{0}\left(r t^{\prime}\right) \int_{0}^{r_{11}} \mathrm{~d} r^{\prime} r^{\prime} J_{0}\left(r^{\prime} t^{\prime}\right)\left[\Theta\left(r_{c}-r^{\prime}\right)+\Theta\left(r^{\prime}-r_{c}\right) \ln \left(r_{11} / r\right) / \ln \left(r_{11} / r_{c}\right)\right]
$$

The weighting potential for our ring detector is shown in Fig. 11.

\section{DISCUSSION}

The carrier collection patterns derived in the preceding section in combination with our main result for the detector response (9), together with the 3D shape of the weighting potential, allow us to interpret the main features of the experimental data presented in Section 3.

\section{A. Hemispherical mode}

The interpretation of the spatial response is straightforward and follows from the carrier collection patterns shown in Fig. 9b. It confirms that as in Fig. 3, the collection region is a cylinder. The explanation of the depression in the center of a circular profile for count rate and peak position is also clear. This occurs because the main contribution to the detector response comes from electrons. In good CdZnTe samples such as the present, the mean electron drift path is much longer than the typical distances to the anode, which for our detector are of the order of $1 \mathrm{~mm}$. Correspondingly, electron trapping is not the most important factor for the count rate, but rather the value of the weighting potential at the point of carrier generation. Comparing electron contributions for generation at the same depth but at different radial distances from the center, we see that electrons generated further away from the center 
(closer to the edges) have lower initial values of weighting potential, as clearly seen in Fig. 11b, and will thus induce greater charge on their way towards the anode. In a real experiment, there exists a distribution of absorption sites in depth, but the same argument holds for any depth, with Fig. 11b serving only for illustration purposes. With poor hole mobility-lifetime product, electrons reaching the collecting anode from the edges make greater contribution to the detector signal than those which were created at the same depth, but closer to the center. On the other hand, holes cannot compensate for the difference in the initial values of the weighting potential, because they are subject to strong trapping. As a result the appearance of a depression in the count rate and the peak position is to be expected. The same factor also plays a dominant role for the resolution. In this detector, the best achieved resolution is limited by the inhomogeneous broadening of the pulse. Indeed, all spectra in the whole energy range of Fig. 6 exhibit an asymmetry which is a direct indication of inhomogeneous broadening. This occurs through the dependence of the collected charge on both lateral and vertical coordinates of the absorption sites. With poor hole transport the maximum inhomogeneous broadening occurs from areas with rapid variation of weighting potential, which according to Fig. 11 are at the center of the detector. For this reason the resolution is best at the edges of response in agreement with experiment.

Although qualitatively we are able to explain all experimental results, there is one important quantitative discrepancy indicating the importance of the CZT surface conductivity as discussed above. Cylindrical collection zone from experimental data extends up to the radius of the second ring. The simulated cylindrical collection zone from Fig.9b has a smaller radius $R$ falling between the inner and outer rings: $r_{12}<R<r_{21}$. By solving the corresponding problem for the detector of finite lateral size we found that this it is not related to the exact boundary conditions on the detector side surface. The only remaining factor which can significantly affect the detector collection pattern is the surface conduction and the resulting distortion of the electric field lines in the vicinity of the gaps between electrodes. Qualitatively we may understand the increase of the collection zone radius compared to the simulated result $R$ using the surface conduction model in CZT described in ${ }^{9}$. For p-type conducting channels at the the gaps between the electrodes, gap size of $100 \mu \mathrm{m}$ and biasing from the Table 1 , the surface channel between the central anode and inner ring is likely to be fully depleted. The stronger lateral deflection towards the center of the detector will cause a shift of the boundary of the cylindrical collection zone bringing it closer to the location of the saddle point of the electrostatic potential in Fig.9a. The latter resides at approximately the radius of the outer ring. 


\section{B. Drift mode}

Spatial profiles observed in Section 3 for operation in the Drift mode can be understood from the collection patterns shown in Fig. 10. For X-rays of $10.7 \mathrm{keV}$, the $1 / e$ absorption depth in CdZnTe is $13.3 \mu \mathrm{m}$. At this depth, taking the section of the collection zone of Fig. 10c by the horizontal line at the level of 0.99 , we see that there will be collection from a central area of approximately $0.2 \mathrm{~mm}$ radius and also collection from the narrow circular ring defined around $0.75 \mathrm{~mm}$, in general agreement with experimental data in Fig. 5 . At 50 keV, the 1/e absorption depth in CdZnTe is $200 \mu \mathrm{m}$ and at the center of the $\operatorname{dip}\left(\simeq 0.4 \mathrm{~mm} \approx 1 / 2\left(r_{21}+r_{22}\right)\right)$ a considerable fraction of electrons falls into the dark area and is not collected by the central anode. But with larger X-ray energy, increasing number of carriers are generated outside the shaded area leading to an increase in collected charge, in agreement with the observations.

Another feature in Fig. 5 can also now be understood. When the X-ray energy increases, the charge collected from the sites at $\simeq\left(r_{21}+r_{22}\right)$ decreases, while the signal amplitude for the central absorptions remains the same (ignoring the distortion due to absorption in the central lead). This occurs because for a focused X-ray beam positioned in the center, the vertical size of collection region is significantly larger than if the X-ray beam is positioned at the $2 R_{2}$ ring center. As a result most of the electrons generated at all depths in the center are still in the light region and contribute to the detector response, while deeper sites for the $2 R_{2}$ position have already fallen in the dark region, so that electrons go to the cathode instead. For a full quantitative description of the data shown in Figs. 6, 7 and 8 , one would require a numerical analysis based on (9), taking full account of the electron and hole transport properties, the distribution of the absorption sites relative to the collecting and non-collecting areas and the 3D shape of the weighting potential. So far we have developed such a model for the detector response combining both analytical and numerical techniques for co-planar grid structures. The extension of the technique to the ring detector would require a fully numerical approach because of the strongly inhomogeneous distribution of electrostatic fields.

The energy resolution of the Drift detector found in the experimental data was slightly better that that for the Hemispherical detector. This can be understood from our model. For all carriers generated laterally at $2 R_{2}$ collection "ring" the magnitude of the weighting potential is less than at any location in the collection area for the Hemispherical mode. Therefore, the Drift mode signal originates to a larger extent from single carrier sensing with less degradation from hole trapping than for the Hemispherical mode. Another feature of the operation in the Drift mode, notably the poor resolution for absorption elsewhere than at $2 R_{2}$ collection ring also finds a simple explanation. As follows from our discussion above, the inhomogeneity in the central areas of the detector leading to the distortion in line 
shape is much stronger for the Drift mode than for the Hemispherical mode. For the latter, it arises only because of poor hole transport properties and rapid variation of the weighting potential. Operating the detector in the Drift mode adds to these factors another source of inhomogeneity, the occurrence of non-collecting zones depending on the lateral position and depth of absorption. This is likely to be the most important factor affecting the line shape and contributing to a much degraded resolution, as observed in experiments.

Narrowness of the simulated carrier collection pattern at twice the radius of the outer ring is likely to occur in our model because of two factors. Firstly, these sites are not very far away from the detector edges. As a result the distribution of radial electric field in their vicinity is likely to be affected by boundary conditions for electrostatic potential at the detector edges. Whilst the effect of edges is negligible for the Hemispherical mode with the collection zone closer to the detector central areas, it is generally stronger for the Drift mode configuration. The second factor is due to stronger electron deflection towards the center of the detector because of the channel depletion between the anode and an inner ring. This will result in the collection zone being wider in the same way as for Hemispherical mode.

\section{SUMMARY}

The present results confirm the potential of the ring-drift concept. In contrast to other detection schemes $^{2}$, it simultaneously gives excellent energy resolution and a wide dynamic range, which makes it particularly attractive in XRF, electron microprobe analysis systems and nuclear medicine applications. The use of a small readout node ensures that electronic noise due to anode capacitance is low and independent of the active detector area, which implies that bigger detection areas can be fabricated with little loss of performance. Additionally, further reduction of leakage current by cooling means that shorter shaping times may be employed, making the device suitable for high count rate applications. We have derived explicit expressions for the detector response in both modes of operation, analyzed carrier collection patterns and showed that all experimental results can be understood within the model approach. The unusual spatial response at lower energies, which suggests that the detector is insensitive over a large fraction of its physical area is an artifact of current detector design, its ring geometry and biasing. Nevertheless, some applications may be able to exploit this attribute; for example, on-axis, small angle scattering or diffraction experiments of the type commonly carried out at synchrotron radiation facilities.

Acknowledgements

We acknowledge V. Ivanov for many helpful discussions. This work was supported by the IHP-Contract II-04-016 
EC of the European Commission. 
1 P.N.Luke, Nucl. Instr. and Meth. A 380 (1996) 232.

2 A. Owens, A.G. Kozorezov, Nucl. Instr. and Meth., A563 (2006) 31-36.

3 A.Kozorezov, A.Owens, A Peacock, J.K.Wigmore, unpublished (APL?)

${ }^{4}$ P. Lechner et al., Nucl. Instr. and Meth., A377 (1996) 346-351.

${ }^{5}$ http://www-hasylab.desy.de/

${ }^{6}$ H.Yoon, J.M.Van Scyos, M.S.Goorsky, H.Hermon, M.Schnider, J.C.Lund, R.B.James, J.Electron. Mater. 26, 529, (1997)

7 A.E.Bolotnikov, W.R.Cook, F.A.Harrison, A.S.Wong, S.M.Schindler, A.C.Eichelberger, Nucl. Instr. and Meth., A432, 326 (1999)

8 T.H.Prettyman, M.K.Smith, P.N.Luke, M.Amman, J.Lee, Proc. SPIE 3768, 27 (1999)

${ }^{9}$ A.E.Bolotnikov, C.M. Hubert Chen, W.R.Cook, F.A.Harrison, I.Kuvetli, S.M.Schindler, C.M.Stahle, B.H.Parker, Nucl. Instr. and Meth., A510, 300 (2003)

10 A.G.Kozorezov, J.K.Wigmore, A.Owens, R. den Hartog, A.Peacock, Journal of Applied Physics, 97 (2005) 074502. 